\title{
Notes sur les Hématozoaires et tiques des animaux domestiques à Madagascar.
}

\author{
por G. UILENBERG \\ (avec une Iıste des Protozoaires, Ricketisiales ef Arthropodes parasites, identifiés dans le Pays.) \\ (Laboratoire Central de l'Elevage de Tananarive)
}

\begin{abstract}
RÉSUMÉ
Les recherches faıtes à Madagascar sur les hématozoaires et rickettsiales du sang des animaux domestiques, sont rapportées.

Une courte discussion montre pourquoi Babesia argentino (Lignières, 1909) est considéré comme nom valable pour la petite espèce bovine à Madagascar.

Pratiquement, tous les bovins adultes, vivant en milieu infesté de tiques, sont porteurs de B. bigemina ; B. argentmo est beaucoup plus rare. La plupart des bovins régulièrement détiqués ne portent pas de Babesia, et c'est surtout sur ces animaux que les babésioses cliniques sont observées. La babésiellose est beaucoup plus fréquente que la piroplasmose vraie, peut-être en partıe parce que B. argentina est rare et la prémunition natureile peu fréquente. La forme cérébrale de la babésiellose est fréquente, celle de la piroplasmose vraie n'a pas été observée. L'acriflavine (Gonacrine) et le quinuronium sulfate (Zothélone) agissent sur les deux maladies ; la pentamidine (Lomidine) est seulement efficace contre la pıroplasmose vraie. La prémunition contre la babésiel lose pose des problèmes parce que $B$. argentina n'est pas régulièrement transmise avec le sang de porteurs chroniques. Les veaux de moins d'un mois sont remarquablement résıstants à l'infection par B. bigemina; une réaction clinique à $B$. argentino est plus fréquente. Lo durée de l'état de prémunition semble souvent être assez courie (moins d'un an).

L'anaplasmose bovine n'est pas fréquenfe. II n'y a guère de bovins adultes, en milieu infesté de tiques, qui ne soient pas porteurs d'A. marginale. Une grande proportion des bovins régulı̀rement détiqués, et dont la plupart ne porte pas de Bobesia, sont néanmoins infestés d'A. marginale. Il semble donc que d'autres vecleurs que les tiques puissent jouer un rôle. Les veaux de moins d'un mols sont très résistants à l'infection par A. marginale. La Terramycine donne satisfaction dans le traitement de l'anaplasmose.

Eperythrozoon wenyoni et $E$. teganodes sont très répandus. Ils ne semblent obéir à aucune règle. Les deux espèces sont apporues sur des veaux splénectomisés, à l'abrı des tiques, et n'en ayant jamais montré pendant les 5 à 8 mois suivant la splénectomıe. Le mode de transmission reste inconnu. Les deux espèces peuvent être pathogènes, même pour des bovıns non splénectomisés, mais aucune mortal ité n'a été observée La néoarsphénamine est efficace.

Un bref exposé est fait sur les trois espèces d'Argasidae et les troıs espèces d'Ixodidae des animaux domestiques à Madagascar. Plusieurs autres espèces de tiques ont été introduites avec du bétail importé, mais ne semblent pas s'être établies dans le pays.

Une liste est donnée des prolozoaires, rickettsiales et arthropodes, parasiles des animaux domestiques à Madagascar, délerminés jusqu'ıcı.
\end{abstract}


Les affections transmises par des arthropodes jovent, comme dans les autres pays tropicaux, un rôle de première importance à Madagascar. Le pays a d'ailleurs le privilège d'être une île, et plusieurs maladies importantes n'ont, de ce falt, pu y pénétrer. Ainsi, on n'y trouve pas de trypanosome pathogène, position heureuse en comparaison de celle de son grand voisin, le continent africain. Le nombre d'espèces de tiques, principaux vecteurs des hématozoaires, parasitant les animaux domestiques, est également faible; toutes ces espèces y ont été importées, les espèces autochtones ne semblanł parasiter que des anımaux sauvages.

Nous ne ferons pas, dans cetfe communication, l'éłude détaillée des infections en question, mais nous tenterons de rassembler les recherches faites d̀ leur sujet à Madagascar. Nous parlerons d'abord de quelques parasites sanguins, en procédant par espèce animale ; ensuite nous ferons quelques remarques sur les tiques des animaux domestıques, et nous terminerons par une liste des protozoaires, rickettsıales et arthropodes parasites des animaux domestıques, déterminés jusqu'icı.

\section{BOVINS}

\section{Genre BABESIA (Starcovici, 1893.)}

Deux espèces ont été trouvées, Babesia bigemino (SMITH et KILBORNE, 1893) et Babesio argentina (LIGNIËRES, 1909), (et non LIGNIERES, 1903, comme l'on trouve dans la plupart des publications ef livres ; voir LIGNIÉRES, 1903 (38) et 1909 (39)).

B. bigemina, cause de la piroplasmose vraie, fut trouvée en 1906 par le vétérinaire Carougeau (cité par BUCK (6), 1934). L'identıté de cette espèce à Madagascar ne fait aucun doute; la morphologie est classique, et la maladie qu'elle cause, correspond à celle décrite ailleurs.

$B$. argentina fut trouvée pour la première fols en 1936 (BUCK, 1937 (8)). Le nom Babesiella berberaSERGENT e. a., 1924, fut d'abordemployé. Plus tard (RAYNAUD, 1962 (56)), ce nom fut changé en Babesıa bovis (BABES, 1888). Nous considérons $B$. argentina comme le nom valable de l'espèce en question. En effet, nous croyons qu'il n'est plus possible de savoir avec certitude à quelle espèce (ou quelles espèces) BABES avalt affaire lorsqu'il décrivait la maladie bovine en Roumanie (BABES, 1888 (3), 1889 (4), CORNIL et BABES, 1890 (21)). Dans cette région d'Europe, quatre espèces de Babesia ont été signalées sur les bovins par la suite, $B$. bigemina, B. divergens (M'FADYEAN et STOCKMAN, 1911), B. major (SERGENT e. a., 1926), et. l'espèce correspondant à celie de Madagascar, B. argentına (SIMITCH e. a., 1955 (64), PAVLOV, 1957 (47), ANGELOVSKI, 1957 (2), CERNAIANU, 1958 (20), PETROVIC, 1958 (48), PETROVIC e. a., 1958 (49) ef KOTLAN e. a., 1959 (36)). De plus, l'existence de plusieurs espèces de tiques dans la région où BABES a étudié la maladie, montre que l'on peut s'attendre à y trouver les quatre espèces de Bobesia (Ixodes ricinus, vecteur de B. divergens, et diverses espèces appartenant aux genres Hoemaphysalis, Rhpicepholus, Hyalomma, Dermacentor et Boophilus, parmi lesquelles figurent des vecteurs prouvés de $B$. bigemina, $B$. argentina ou B. berbero, et B. major; FEIDER e. a., 1958 (25 et 26).

If nous semble donc que Davies e. a. 1958 (23 \& 24) ont plus ou moins par hasard obtenu une souche en Yougoslavie de l'espèce qui correspond à celle dont il est question, et que nous appelons B. argentina. Nous croyons qu'ils auralent aussi bien pu recevoir une souche de B. divergens, même de B. major, pour leurs comparaisons d'une petıte espèce de Yougoslavie à $B$. divergens de la Grande-Bretagne.

Nous estimons préférable de ne plus utlliser le nom Babesia bovis, puisque Babes ne savait pas qu'il $y$ avait plusieurs espèces du genre, et puisque ce nom prête d̀ confusion ; nous préférons employer des dénominations sans équivoque : l'espèce européenne, trouvée jusqu'aux parties septentrionales de l'Europe, appelée pendant longtemps par la plupart des auteurs $B$. bovis est $B$. divergens, et l'espèce décrite par LIGNIÉRES, appelée $B$. bovis par SIMITCH et NEVENITCH, 1953 (63), par DAVIES e. a., 1958 (23 \& 24) et par d'autres après ces quteurs, est B. argentina.

Nous considérons avec RAYNAUD, 1962 (56), et pour les mêmes raisons que lui, que le nom de $B$. berbero est synonyme de $B$. bovis (sensu SIMITCH et NEVENITCH), et nous considérons donc $B$. berbera comme synonyme de $B$. argentina.

$B$. argentina à Madagascar correspond à la description qu'en ont donné les divers auteurs 
dans d'autres pays (de B. argentına, B. berbera ou B. bovis sensu SIMITCH ef NEVENITCH), tant en ce qui concerne sa morphologie, sa distribution dans le corps de l'hôte, que le tableau clinique de la maladie qu'elle cause.

\section{GÉNÉRALITÉS \\ SUR LES BABESIOSES BOVINES A MADAGASCAR}

Leur pathogénie est identique à celle décrite dans d'autres pays où ces hématozoaires provoquent des enzooties. Les cas de maladie clinıque sont rares parmi les immenses troupeaux de zébus malgaches, tant qu'ils ne sont pas détiqués. Les animaux importés succombent rapidement, s'ils ne sont surveillés et soignés très attentivement. Les veaux, nés dans le pays en milieu infesté de tiques, montrent une résistance considérable aux infections et ne présentent, le plus souvent, pas de symptôme clinique ou font une légère maladie (cette résistance est surtout remarquable envers $B$. bigemina) ; ils se prémunissent par'les piqûres de tiques, et la prémunition estentretenue par des réinfections contınuelles par ces tiques. Cette résistance des jeunes veaux n'existe pas seulement chez les zébus malgaches, mass également chez les races bovines importées. Une résistance associée à l'âge intervient certainement dans ce phénomène, mais une immunité passive, transmise par la mère, pourralt également jouer un rôle, comme pour $B$. argentina (HALL, 1960(31)). Dans les élevages ou les bovins sont régulièrement détiqués, les zébus malgaches peuvent également présenter des cas clınıques souvent mortels, tout au moins en ce qui concerne B. argentina (Buck et Metzger, 1940 (15)).

\section{TRANSMISSION}

La transmission des deux espèces de Babesia par Boophilus microplus a été prouvée expérimentalement à plusieurs reprises par notre confrère RAYNAUD et par nous-mêmes. B. microplus est ubiquitaire dans le pays, ce qui explique la grande diffusion des infections à Babesia.

\section{LA MALADIE}

B. bigemino: Rien de particulier n'est à signaler en ce qui concerne les symptômes de la piroplasmose vraie à Madagascar.
L'incubatıon parasıtaıre (c'est-à-dıre, le délai jusqu'au premier jour où le parasite est trouvé sur frottıs de sang), après infestation par des larves de B. microplus est de 13,14 et 17 jours (RAYNAUD, Rapport Annuel du Laboratoire de 1961). Nous avons trouvé, chez des animaux sur lesquels des frottıs de sang ont été faits tous les jours, une incubation parasitaire, après inoculation de sang infecté, de 5, 6, 7, 9 et 9 jours sur des bovins non splénectomisés, et de $7,7,7$ et 9 jours sur des animaux splénectomisés. L'incubation thermique (c'est-à-dire, le délai jusqu'au premier jour de l'hyperthermie) a été de 11 jours sur un des anımaux non splénectomisés (les autres ne faisant pas de réaction thermique) et de $8,9,9$ et 10 jours sur les splénectomisés. (Observations non publiées.) L'ablation de la rate ne semble donc pas avoir d'influence sur la durée de l'incubation parasitaire. L'incubation thermıque a toujours été d'un à trois jours plus longue que l'incubation parasıtaire.

En nous basant sur des splénectomies effectuées sur plusieurs bovins adultes en provenance de différentes régions de l'île (RAYNAUD, 1962 (56) RAYNAUD et UILENBERG, 1962 (59)) et sur des inoculations de sang de bovins adultes que nous avons effectuées sur des veaux sensibles, il semble que pratiquement tous les bovins adultes vivani en milieu infesté par des B. microplus, sont porteurs de B. bigemino. Ceci n'est pas étonnant, étant donné la grande diffusion de cette tıque dans le pays. II n'en est pas de même pour les anımaux régulıèrement détiqués, RAYNAUD, 1962 (56) n'a pu observer une seule infection à $B$. bigemina sur 16 bovins splénectomisés, en provenance du Centre de Recherches Zootechnıques de Kianjasoa, où tous les animaux sont régulièrement passés au bain détiqueur, et où nous n'avons pas observé de B. microplus, bien que la tıque solt très fréquente dans la région autour du Centre. Nous avons plus tard splénectomisé encore 7 bovins du Centre ef n'avons pu observer la sortie de B. bigemina que sur 2 d'entre eux.

B. argentina : L'incubation parasitaire, après l'infestation par des larves de B. microplus, observée por RAYNAUD (Rapport Annuel de 1961) a été de 27 jours; nous avons observé des périodes de 10,16 et 16 jours. (L'incubation thermique était également de 10,16 et 16 jours.) L'incubation parasitaire après inoculation de 
sang infecté a été de $11,11,11,12,13,14,16$ et 17 jours, ef l'incubation thermique de 10,11, 11, $11,11,14,16$ et 17 jours (observations non publiées). (La période d'incubation parasitaire observée n'est certainement pas toujours exacte, puisque les parasıtes sont si rares dans le sang périphérique, qu'on peut facilement ne pas les trouver pendant quelques jours.)

La maladie causée par B. argentina, la babesieliose, se caractérise par, la localısation des protozoaires dans les organes Internes; ils sont toujours rares dans le sang périphérique. Le diagnostic est plus facile après la mort, sur frottis de cortex cérébral et sur calques de rein, rate et foie. On trouve les capillaires du cortex cérébral bourrés d'érythrocytes parasités et le rein, la rate et, à un moindre degré, le foie en contiennent également un grand nombre. Lo forme cérébrale nous semble plutôt la règle que l'exception. Nous n'avons, par contre, jamais observé, avec certitude, la forme cérébrale de la piroplasmose vraie.

La babésiellose est une maladie plus grave que la piroplasmose vraı et plus difficile à traiter. Le parasite semble d'ailleurs beaucoup moins répandu que $B$. bigemina et pourrait être un hématozoare d'importation (RAYNAUD et UILENBERG, 1962 (59)), bien que, plus tard, nous l'ayons vu sur des prélèvements en provenance de plusieurs endroits de l'île, autres que ceux mentionnés dans la publication précitée. Il est possible que la moındre fréquence de $B$. argentino dans le pays solt une des rasons pour lesquelles la babésiellose-maladie esł beaucoup plus fréquente que la piroplasmose-maladie, pusque moins d'animaux possèdent l'état de prémunition contre B. argentina.

\section{LUTTE CONTRE LES BABESIOSES}

Nous disposons de trois moyens pour combattre ces maladies :

10 Traitement des malades.

20 Lutte contre le vecteur B. microplus.

3o Prémunition artificielle des animaux sensibles.

\section{Traitement.}

Des essais de traitement avec divers piroplasmicides sont en cours, principalement sur des animaux splénectomisés, ce qui constitue une épreuve sévère, puisque les animaux faisant des rechutes après splénectomie meurent presque toujours, s'ils ne sont pas traités (il $y$ a eu des exceptions pour B. bigemina, qui quelquefois ne fait qu'une apparition fugace, ne nécessitant pas d'intervention thérapeutique) ; les animaux splénectomisés, indemnes de Babesia, ont toujours fait une réaction sévère aux deux parasites, mortelle en l'absence de traitement, après infection par du sang ou des tiques.

B. bigemina : Le traitement ne pose pas de problème. Tous les piroplasmicides expérimentés se sont montrés efficaces et une seule injection a presque toujours suffi. Nous avons, jusqu'ici, essayé le trypanbleu, le quinuronium sulfate (Zothélone), la pentạmıdine (Lomidine), I'acriflavine (Gonacrine) et le di-iséthionate d'amicarbalide (Pirodia). Les indications des fabricants ont été suivies quant au dosage. Nous utilısons le trypanbleu à dose faible $(0,4$ à $0,6 \mathrm{~g}$ par animal de 100 à $200 \mathrm{~kg}$ ); nous n'avons pas observé de souche résistante à ce médicament, comme I'a fait notre confrère RAYNAUD, 1962 (57).

Nous préférons la Lomidine, efficace, facile à admınistrer (voie intramusculare) et, dans notre expérience, sans réaction secondaire. Le trypanbleu et la Gonacrine sont efficaces, mais doivent être injectés par vole intraveineuse, difficile en brousse. Le Zothélone est facile à administrer (en sous-cutanée) et efficace, mais son empla est délicat, les réactions secondaires étanł fréquentes et quelquefois mortelles (tout au moins sur les splénectomisés). Nous n'avons pas encore assez d'expérience du Piradia pour donner une opinion fondée; son emplo est faclle (en souscutanée) et il a été efficace et sans réaction secondaire sur le premier veau expérimenté.

B. argentina : Le traitement de la babésiellose est beaucoup plus difficile. Il, est certain que quelques piroplasmicides agissent sur le parasite, mais l'administration doit être précoce. comme l'ont déjà indiqué BUCK et METZGER, 1940 (15). Nous ne pouvons pas être d'accord avec TSUR, 1961 (72), qui estime que ce protozoaire (appelé $B$. bovis) n'est pas influencé par la chimiothérapie; nous réussissons même à guérir les splénectomisés.

D'après tous les auteurs, le trypanbleu n'agit pas, aussi l'utilisons-nous pour traiter des accès de $B$. bigemina dans les cas d'infection mixte, où 
nous ne voulons pas influencer B. argentina par le traitement. La Gonacrıne et le Zothélone sont actifs, ainsi que nous avons pu le constater à plusieurs reprises, ef comme l'ont déjà indiqué BUCK et METZGER, 1940 (15) (La Gonacrine nous semble quelque peu plus efficace que le Zothélone). La Lomidine n'a pas montré une efficacité satisfalsante. Nous n'avons pas encore pu expérimenter d'autres produits.

Dans la pratique, sans microscope, il est, le plus souvent, impossible de savoir à quelle espèce on a affaire; aussi le tratement doit-il être polyvalent, c'est-c̀-dıre que, parmi les prodults expérimentés jusqu'ici, seuls la Gonacrine ef le Zothélone semblent convenir. Ces produits ayant des inconvénients (administration par voie intraveineuse pour l'une et réactions secondaires pour l'autre), nous continuons les recherches sur le traitement des babésioses. II est très important que le traitement ne stérilise pas l'animal, c'est-à-dire qu'il laisse subsister l'état de prémunition après guérıson.

\section{Lutte contre le vecteur.}

Dans les circonstances actuelles, il nous paraît impossible d'obtenır l'élimination de Boophilus microplus à Madagascar, et nous croyons qu'il serait souhaitable de maintenir dans les élevages une légère infestation par cette tique, qui, sans porter afteinte à la santé des animaux por ses effets directs, entretiendrait un état de prémunition naturelle dans le cheptel. Malheureusement, la question est compliquée par la présence de la heart-water et son vecteur. Amblyomma variegatum, dans la plus grande partie du pays ; la lutte contre les tiques doit, de ce fait, être menée de façon vigoureuse, tout au moins dans les élevages possédant des bovins de race importée ou améliorée. De plus, la streptothricose cutanée est un grand problème dans les régions plus ou moins basses et chaudes; le meilleur moyen de prévention contre cette maladie semble être les bains réguliers d'arsenic, ce qui constitue en même temps un bon détiquage.

Pour l'instant, nous sommes donc obligés de conseıller un détıquage très efficace, avec le risque de vorr des cas de babésiose se produire sur les animaux non prémunis, lors de l'introduction accidentelle de tiques ( $c$ 'est ce que l'on observe en effet) ; cet état de choses pourrait changer lorsque nous pourrons vacciner contre le heart-water, et lorsqu'un médicament efficace contre la streptothricose ne possédant pas de qualités ixodicides, sera trouvé.

Prémunition artificielle des animaux sensibles

Les animaux vivant en milieu non détiqué, sont prémunis pendant les premiers mois de leur vıe grâce aux B. microplus. Cette prémunition est entretenue par les tıques. Aussi, la prémunition artificielle est-elle superflue pour ces animaux. Les cas cliniques de babésiose sont presque toujours constatés dans les élevages où l'on détique régulièrement; l'intróduction accidentelle du vecteur dans l'enceinte de l'élevage (bovins des environs qui s'y égarent, achał d'animaux porteurs de tiques, etc...) déclenche, chez ces animaux non prémunis de façon naturelle, des cas de maladie grave. Aussı peut-on envisager, dans ces conditions, la prémunition artificielle. Elle pose d'ailleurs de nombreux problèmes.

La surveillance et le traitement éventuel de la réaction doivent être assurés; ils ne le sont souvent pas, par négligence des propriétaires, ou par manque de personnel dans le cas des grands centres possédant des centaines d'animaux.

Nous avons fait quelques expériences sur la prémunition des jeunes veaux, dans le but de voir sı leur résistance est suffisamment grande pour ne pas exiger de survellance. La dose de sang inoculé (par voie sous-cutanée) a été de $10 \mathrm{cc}$; le sang provenait de bovíns splénectomisés, porteurs chroniques de $B$. bigemina et $B$. argentina.

Envisageons, d'abord, la réaction vis-à-vis de B. bigemina.

Sur 5 veaux de moins d'un mois, dont la température et des frottis de sang ont été pris tous les. jours, un seul a fat une réaction sévère à B. bigemina ef a été traité ; les autres ont tous montré le parasite dans le sang, mass il n'y avait pas de réaction clinique ef le traitement n'a pas été nécessaire. Sur 13 veaux, dont 12 avaient moins d'un mois ef l'autre presque deux mois, dont la température a été prise tous les jours par le propriétaire ef dont des frottis de sang ont été examinés une fois par semaine, aucun n'a 
fait de réactıon clinique à $B$. bıgemına nécessitant un traitement; le parasite est apparu dans le sang sur 10 de ces animaux, mais il est possible que les trols autres aient fait leur crise parasitaire entre les examens hebdomadaires du sang (ou bien, étaient-ıls déjà prémunıs, ce qui est peu vrasemblable, les veaux vivant dans des élevages détıqués efficacement). Les vequx utilisés étaient de race frisonne et normande, quelqus-uns ayant une légère proportion de sang zébu ou rana (bovins indigènes, descendant de races européennes importées anciennement).

Un autre veau (normand) a été inoculé avec du sang de bovin en période d'incubation de B. bigemina, qui apparaissait quelques jours plus tard dans son sang et lui causait une maladie grave. Le veau inoculé avec ce sang fut infecté par B. bigemina ef fit une réaction sévère, nécessitant un traitement. Si l'on exclut ce veau du résultat de l'expérience (puisque le fait que le donneur était en incubation a pu avoir de l'influence sur la virulence du protozoaire), nous pouvons conclure que sur 18 vequx, dont 17 de moins d'un mors et un de presque deux mois, inoculés avec $10 \mathrm{cc}$ de sang de bovins splénectomisés, porteurs chroniques de B. bigemina, un seul a fait une réaction grave, tandis que dans 15 cas, il fut démontré que $B$. bigemina a été transmise.

Les résultats de l'expérience sont incertains en ce qui concerne $B$, argentina. 5 des 19 veaux ont fait une réaction importante et ont dû être traités. Le parasite n'a été trouvé que sur ces 5 veaux. Nous ne pouvons donc pas être sûrs de l'avoir transmis aux autres. Cet hématozoaire est toujours rare dans le sang périphérique : un animal peut le montrer dans le sang pendant un ou quelques jours lors de la crise clinique, mais, après guérison, on ne le retrouve presque plus jamais sur les porteurs chroniques ; même lors des crises cliniques on ne trouve pas toujours le parasite. Nous n'avons pas réussi à transmettre expérimentalement $B$, argentina à deux veaux splénectomisés, indemnes de ce protozoaire, avec $10 \mathrm{cc}$ de sang d'un veau (non splénectomisé), qui 2 semaines avant, avait falt une légère crise clinique avec frottis positifs pendant 3 jours : $50 \mathrm{cc}$ de sang du même veau, pris un mois après sa crise clinique et parasitaire, n'ont même pas infecté les deux veaux splénectomisés.
La méthode de MAHONEY, 1962 (42), utilisant le test de la fixation du complément, pourrait peut-être apporter une aide importante pour vérifier si $B$. argentino a été transmıse ou non à un animal donné.

En conclusion, la surveillance de la réaction après inoculation de sang contenant $B$. bigemino et $B$. argentino est indispensable, même lorsqu'il s'agit de jeunes veaux, $B$, bigemina est régulıèrement, ou presque régulièrement, transmise par une dose de $10 \mathrm{cc}$, en sous-cutanée, de sang de porteurs chroniques splénectomisés; nous ne croyons pas que ce soit le cas pour'B. argentina (voir aussi TSUR, 1961 (72) ef TSUR et LAPINSKI, 1962 (73)). Mals la babesiellose est justement la maladie la pius grave et, comme maladie clinique, beaucoup plus fréquente que la piroplasmose vraie. Nos recherches sur la prémunition contre la babésiellose seront donc poursuivies.

Un autre problèmé est posé par la durée de l'état de prémunition. En milieu non détiqué, la prémunition est entretenue par les tiques. Mais, dans les élevages où les $B$. microplus sont rares ou absents, la prémunition peut disparaître au bout d'un certain temps. SERGENT e. a., 1945 (62) indique la durée de l'infection latente à $B$. bigemino comme étant supérieure à 2 ans, et de $B$. argentina (B. berbera) supérieure à 1 an. NEITZ, 1962 (46) rapporte par contre deux cas où la prémunition à $B$. bigemina ne persistait plus après respectivement 3 et 8 mois. RIEK, 1962 (61) signale que les infections à $B$. bigemina peuvent durer plus de 2 ans, mais que certains animaux peuvent être négatifs après un an: toujours d'après luI, B. argentina peut disparaître en 66 jours, mais persiste en général de 12 à 18 mais. Nous avons signalé (Rapport Annuel 1962) un cas où un veau avait perdu l'infection à B. bigemina en moins de 10 mois; récemment encore nous avons pu constater; sur deux autres animaux, que l'infection à $B$. bigemina avait disparu respectivement en moins de 8 mois et $1 / 2$ ef en moins de 11 mois. (Nous considérons comme preuve le fait que nous n'avons pas réussı à transmettre $B$. Bigemina avec leur sang d̀ des veaux sensibles, et qu'une inoculation de sang infecté a déclenché, chez les anımaux en question, une réaction parasitaire et, dans deux des trols cas, une maladie grave.) Par contre, un autre veau hébergeait $B$. bigemina 5 mois et $1 / 2$ 
après son infection, comme nous l'a montré sa splénectomie.

Eu égard au fable nombre d'animaux éprouvés, il nous semble qu'une durée relativement courte de la prémunition soit plutôt la règle qu'une exception. Si cela se confirmait par la suite, la prémunition artificielle deviendrait encore plus difficile, puisqu'il faudrait la refaire au moins tous les ans sur les animaux dans les élevages où l'on pratıque un détiquage efficace ; tous les animaux devraient être surveillés chaque fois, ce qui est difficile à réaliser dans les grands centres.

Genre THEILERIA Bettencourt, França et Borges, 1907.

Seule l'espèce Theileria mutons (THEILER, 1906) a été trouvée dans le pays. On falt état de son existence à partir du Rapport Annuel de 1932. Aucun cas de maladie n'a jamass été atțribué à Th. mutons à Madagascar. Le parasite est même pratiquement apathogène pour les animaux splénectomisés. Nous avons quelquefois traité des veaux splénectomisés qui avalent depuis longtemps une infestation sanguine massive, et qui étaient chroniquement en assez mauvais état, ce que nous croyons pouvorr imputer à Th. mutons. Le médicament employé a été l'Antimosan, qui semble avoir eu un certann effet sur le parasite (voir aussı RAYNAUD, 1962(58)). D'autres, bovins splénectomisés, présentant des infestations aussı massives et en aussi mauvais état, n'ont pas été traités et ont, peu à peu, repris un état normal en même temps que la parasitémie diminuait.

On peut pratiquement foujours trouver ce protozoaire dons le sang des bovins infectés, même lorsqu'll s'agit d'animaux non. splénectomisés, bien qu'il soit souvent nécessaire de chercher plus longtemps chez ces derniers. Nous n'avons jamais vu la disparition du parasite sur les animaux infectés, pendant des périodes d'observation allant jusqu'c̀ 2 ans.

Le made de transmission n'est pas connu à Madagascar. RAYNAUD (Rapport Annuel 1961) n'a pas réussi à transmettre Th. mutons par $B$. microplus, toutcomme CALLOW etHOYTE, 1961 (18) en Australie. II n'est guère de bovins à Madagascar qui n'en solent pas porteurs, ainsı que l'ont montré de nombreuses splénectomies (RAYNAUD, 1962 (56 \& 58), RAYNAUD et UILENBERG, 1962
(59), ef d'autres plus récentes, dont les résultats n'ont pas été publiés). Le parasıte est mêrne trouvé sur la plupart des veaux en provenance des centres où le détıquage est efficace, et où la plupart des animaux ne portent pas de Babesia. Il iest aussi fréquent dans les régions ou Amblyomma voriegatum et Rhipicephalus sanguineus sont absents ou très rares (Cette dernière tique ne se rencontre d'ailleurs qu'exceptionneilement sur les bovins à Madagascar ; personnellement nous ne l'avons jamaıs trouvée que sur des chiens). Nous n'avons pas pu consiater de transmission intra-utérine sur 9 veaux qui ont été contrôlés à la naissance et ont pu être suivis.

Nous avons observé, après inoculation de sang contenant des formes érythrocytaires, des pérıdes d'incubation parasitarre de 13, 16, 17. $17,30,31$, et 33 jours (Les parasites étant au début très rares dans le sang, il est, certain que l'ıncubation parasitaire est, en réalité, pluș courte).

\section{Genre ANAPLASMA Theller, 1908.}

Bien que ces micro-organismes ne font pas partie des Protozoo, ils sont le plus souvent étudiés avec les hématozoaires.

\section{Anaplosmo marginale Theller, 1910.}

La maladie causée par ce parasite, l'anaplasmose bovine, fut signalée à Madagascar pour la première fols par, CAROUGEAU (1913) (19).

Les splénectomies effectuées par notre Confrère RAYNAUD et par nous-mêmes (vorr les publications citées plus haut), montrent qu'il n'y a guère de bovins adultes à Madagascar qui ne portent pas A. marginale. Tous les animaux en provenance de milieu non détıqué en éłaient porteurs; en ce qui concerne les Centres, où le détıquage est efficace, une grande proportion des animaux sont néanmoins infectés d'A. marginale, tout en étant indemnes de Babesia. $\mathrm{Ce}$ résultat est surprenant, vu la facilıté avec laquelle nous avons pu transmettre les Babesiae par $B$. microplus et le fait que RAYNAUD (Rapport Annuel 1961) n'a pu transmettre A. marginale par cette espèce de tique. II faut dire que la période d'observation de RAYNAUD a pu être insuffisante (THEILER 1912 (68) ef HENNING, 1956 (32) indiquent que la période d'incubation 
après transmission par des tiques peutaller jusqu'c̀ 100 jours et plus). D'autre part, il est possible que latransmission d'A. marginale dans les Centres soit aidée par les vaccinations annuelles contre le charbon, avec du sang adhérent d̀ l'aiguille. Il a été prouvé, surtout aux Etałs-Unis, que la transmission de l'anaplasmose par les insectes piqueurs est Importante (vorr PIERCY, 1956 (50)). II nous semble possible que les insectes piqueurs, et peut-être les vaccinations périodiques, soient aussi importants pour la transmission de i'anaplasmose à Madagascar, que les tiques, maıs des recherches sont à faire pour préciser ce point.

Nous n'avons pas vu de transmission intrautérine sur 9 veaux, contrôlés dès la naissance.

La maladie clinique est assez rare, malgré, et sans doute grâce à, l'ubıquité du parasite. Ceci peut être expliqué par des infections naturelles (par des tiques ou des insectes piqueurs?) des jeunes veaux, qui ont alors une grande résistance. Nous avons éprouvé cette résistance des veaux :

18 vecux, dont 17 de moins d'un mois et un de presque deux mois, ont été inoculés avec $10 \mathrm{cc}$ de sang de porteurs chroniques, splénectomisés, de souches virulentes d'A. marginale (par voie sous-cutanée). (Il s'agıt des mêmes veaux et des mêmes donneurs que ceux utilisés dans les expériences sur les Bobesiae; les donneurs étaient porteurs des deux Babesioe et d'A. marginale.) Le parasite est apparu dans lesang de tous. Sur 13 le nombre d'hématies infestées est resté faible, sur 5 le nombre était plus important. Aucun des veaux $n$ 'a réagi cliniquement et sur aucun le nombre d'A. morginale n'est devenu assez important pour nécessiter un traitement. Sur 4 veaux nous avons observé des lésions d'anémie plus ou moins prononcée (en particulier basophilie et polychromatophilie), mais il n'était pas toujours possible de savorr sı ces lésions étaient dues à A. marginale ou à des accès de Bobesio ou d'Eperythrozoon.

Il semble donc possible de prémunir les jeunes veaux contre l'anaplasmose par l'inoculation d'une souche virulente $d$ 'A. marginale, sans qu'aucune surveillance de la réaction solt nécessaire (Toutefois, la mort d'un veau métis normand-zébu, àgé de 48 jours, dont des prélèvements ont été reçus en 1963 au Laboratoire, ef dans le sang duquel nous avons trouvé de nombreux $A$. morginale, doit inciter à la prudence : d'ailleurs, le sang de cet animal contenait également de rares $B$. argentino et $B$. bigemina et if est possible que la babesiellose ait joué un rôle).

Les bovins plus âgés peuvent être prémunis par I'inoculation d'Anaplasma centrale THEILER, 1911, dont nous avons reçu une souche en 1963, grâce à l'amabilité du Dr :TSUR d'Israël (La souche reçue quelques années plus tôt du Dr NEITZ d'Onderstepoort, avait été perdue ;'la bluetongue existant en Afrique du Sud, et non à Madagascar, le Dr NEITZ nous a conseillé d'importer une souche d'A. centrale d'un autre pays).

L'incubation parasitaire, après inoculation de sang, est difficile à préciser, le parasite étant très rare dans le sang au début. Nous avons trouvé les périodes suivantes (premier jour où l'Anoplasma était déterminé avec certitude dans le sang) :

A. marginale : 16, 19, 21, 21 ef 24 jours (Veaux non splénectomisés).

A. cenfrale : $5,7,9,10$ et 20 jours (Veaux splénectomisés. Dans les 4 premiers cas, il s'agissait de doses massives de sang d'un donneur en pleine crise parasıtaire, ce qui explıque la très courte durée de l'incubation. Le veau ayant une incubation parasitaire de 5 jours, avait une hyperthermie de $40,1^{\circ} \mathrm{C} 15$ jours après linoculation, coincidant avec un taux très élevé d'hématies parasitées).

A. centrale a été utilisé depuis quelques années à Madagascar pour la prémunition de plusieurs centaines de bovins de tous âges ; on n'a pas signalé de réaction, sauf dans une ferme de Tuléar, où 6 des 61 bovins inoculés ont présenté une hyperthermie, 8 à 18 jours après l'injection, 5 de ces 6 animaux sont morts après des périodes de maladie variant de 4 à 23 jours. Nous avons eu l'occasion d'examiner des prélèvements de 3 des 5 animaux morts, et de diagnostiquer la babésiellose sur un d'entre eux (mort 4 jours après le début de l'hyperthermie); nous n'avons pas trouvé d'anaplasmes sur ces 3 anımaux. Nous croyons pouvoir exclure la transmission de B. argentina par le sang de notre donneur, veau splénectomisé, qui n'a jamais présenté de maladie imputable aux Babesioe ef $n^{\prime}$ 'a jamais été traité avec un piroplasmicide. II serait mort de Babésiellose s'il avait été porteur avant sa splénectomie ou infecté accidenteilement après. II était d'ailleurs connu que la babésiellose existait déjà à l'a ferme en question. 
Pour la prémunition avec $A$. centrale, nous: nous en tenons aux consells donnés par TSURTCHERNOMORETZ, 1959 (74), qui ne vaccine pas les vaches en gestation avancée, ni celles en pleıne lactation.

A. centrale est même peu pathogène pour les veaux splénectomısés, malgré le fait que l'infestatıon sanguine puisse devenir très élevée. Un seul veau splénectomisé a eu une hyperthermie de $40,1^{\circ} \mathrm{C}$ avec une très forte infestation des hématies, ef nous l'avons traité; nous ne savons pas s'il aurait guéri sans traltement.

Le médicament utılısé à Madagascar dans le traitement de l'anaplasmose est surtout la Terramycine, efficace même dans les cas aigus, quand elle est employée assez tôt. Le Spirotrypan a été essayé sur deux animaux, ayant une assez forte infestation du sang, mais sans hyperthermie: le produit n'a pas, ou tout au plus extrêmement lentement, influencé le taux de parasitémie: nous n'avons pas encore eu l'occasion de l'essayer sur des cas clinıques.

\section{Genre EPERYTHROZOON Schilling, 1928.}

Ces organismes sont classés dans la famille des Bartonellaceae, de l'ordre des Rickettsiales, mais nous les mentionnons $\mathrm{icl}$, puisque ce sont des parasites du sang, qui interviennent fréquemment Jors des expériences sur les hématozoaires.

L'existence d'Eperythrozoon sur les bovins de Madagascar fut découverte par notre confrère RAYNAUD, à la suite d'une splénectomie (Rapport Annuel, 1960). Plus tard, il a été reconnu que ces micro-organismes sont très fréquents dans le pays (RAYNAUD, 1962 (56 \& 58), RAYNAUD ef UILENBERG, 1962 (59)). A cette époque, une seule espèce était connue, $E$. wenyon $A D L E R$ et ELLENBOGEN, 1934. HOYTE, 1962 (35) a séparé un autre parasite de cette espèce et le nommait $E$. teganodes. Nous avons pu voir par la suite que les deux espèces existent à Madagascar. et y sont très répandues. Nous acceptons provisoirement l'opinion de HOYTE, pour qui il s'agit de deux espèces différentes, 'avec quelques réserves pourtant, puisque ce sont des parasites capricieux, difficiles à expérimenter. En fait, il ne semble y avoir aucune règle pour l'incubation et les rechutes. Tantôt c'est $E$. teganodes qui fait le premier son apparition après splénectomie ou inaculation de sang infectieux, tantôt c'est
E. wenyoni. Parfois les accès parasitaires sont très importants, d'autres fois ils restent lımités à de rares parasites.

Le mode de tronsmission des Eperythrozoon bovins est inconnu. Les deux espèces sont apparues sur trols veaux, 5 d̀ 8 mols après leur splénectomie et l'ınoculation de sang contenant A. centrole. Nous ne pouvons pas expliquer cette apparition soudaine. Nous croyons pouvoir exclure l'intervention de tiques. B. microplus est, certes, très commun à Tananarive, mais les veaux en question sont logés dans une étable à l'abri des tiques et ils sont soigneusement détiqués deux fais par semaine par douchage à 0,4 p. 100 de Sevin (Des expériences non publiées nous ont montré, que la protection contre les larves de B. microplus est, à ce pourcentage, au moins de 7 jours, et à 0,1 p. 100 au mons de 4 jours). A. variegatum est très rare à Tananarive $: R h$. sanguineus ne semble pas $y$ exister (De plus le Sevin est actif contre ces tiques). II ne nous semble pas probable non plus. que les puces et les poux (tous deux très communs) puissent être accusés, le Sevin'étant actif contre ces insectes. Il y a la possibilité de transmission par d'autres insectes (le Sevin est peu actif contre les mouches par exemple), ou une transmission accidentelle, lars de la confection des frottis de sang, etc... ; mais nous n'avons jamass encore eu, de telles transmissions d'autres espèces de parasites sanguins, bien que le manque d'espace nous force à loger ensemble des anımaux ayant différentes espèces d'hématozoaires.

Les différentes durées d'incubation d'E. wenyoni et $d ' E$. tegonodes, que nous avons observées après les inoculations ou les splénectomies, et le falt que les deux espèces provoquent, le plus souvent, des rechutes à différentes époques, semblent confirmer qu'ıl s'agıt bien de deux espèces différentes. Cette opınion est renforcée par le fait que nous avons pu transmettre $E$. teganodes à un veau qui étaıt déjà porteur d'E. wenyon. La morphologie des deux espèces semble qussi, quelque peu différente même quand on ne considère pas leur position en rapport aux érythrocytes. On trouve souvent des anneaux d'E. wenyoni libres entre les hématies, surtout dans la queve d'un frottis (ef sans doufe par la confection de l'étalement de sang); ces anneaux sont moins nets que ceux d'E. tegonodes. Nous n'avons jamais observé d'infestations à $E$. teganodes, sans qu'il y 
ait de rares formes accolées à la périphérie des globules rouges: mais ces formes prennent une coloration moins sombre que lorsqu'il s'agit d'E. wenyoni.

Le tableau total des Eperythrozoon bovins est tellement confus, qu'il nous semble toutefois nécessaıre d'être prudent quant à la validité d'E. teganodes.

Actuellement, nos donneurs d'A. centrale sont contaminés des deux Eperythrozoons (il s'agit des trois vecux dont nous avons parlé plus haut). $\mathrm{Ce}$ fait est gênant pour la prémunition, puisque les deux espèces peuvent causer de la fière et de l'anémı plus ou moins prononcée, même chez des bovins non splénectomisés, comme nous l'avons vu à plusieurs reprises; les anımaux guérissent d'ailleurs toujours en l'absence de traitement, et il n'y a eu aucune mortalıté. Pas de mortalité non plus sur des bovins splénectomisés, bien que nous ayons, le plus souvent, laissé l'évolution des accès suivre son cours naturel. Les symptômes observés ne sont pas caractéristıques, ils sont semblables pour les deux espèces ; le symptôme essentiel est la fièvre, quelquefois plus de $40^{\circ} \mathrm{C}$, sulvie par des lésıons d'anémie sur les frottis de sang i les animaux ne parassent pas très abattus.

La néoarsphénamıne agit de façon spécifique. $1 \mathrm{~g}$ par $100 \mathrm{~kg}$ coupe les accès; dans le cas $d^{\prime} E$. tegonodes, nous n'avons jamais retrouvé de parasites le lendemain du traitement; l'action sur E. wenyon est également marquée, mais nous avons vu que quelques parasites peuvent persister ef l'on peut parfois observer des rechutes peu de temps après. Le trypanbleu, I'Auréomy. cine, la Terramycine, la pénicilline, la streptomycine, le Zothélone, la Gonacrine, la Lomidine, semblent sans action contre les Eperythrozoons (observations fortuites, lorsque le traitement d'un autre hématozoaire au d'une maladie coincidait avec un accès d'Eperythrozoon).

II n'a pas été observé de cas d'éperythrozoonose naturelle, à Madagascar.

Nous ne parlerons pas ici de la rickettsie Cowdria ruminantum (Cowdry, 1925), cause de la heart-water, que l'on ne saurait classer parmı les micro-organismes véritablement parasites du sang.

Mentionnons que nous avons vu des spirochètes, ayant la morphologie de Borrelio thelleri (LAVERAN, 1903), dans le sang d'un bovin en
1963 ; nous avons pu le transmettre par inoculation de sang à deux autres bovins ; nous n'avons pas observé de symptôme clinique, et le spirochète ne semble pas perturber les expériences avec les hématozocires.

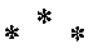

\section{EQUIDAE}

Babesia caballi (NUTTALL, 1910) et Babesia equi (LAVERAN, 1901) ont été signalées à Madagascar.

(Babesia equi est souvent appelée Nuttallia equi ou Babesia (Nuttalia) equi ; en effet, par le falt de sa division en quatre éléments piriformes au lieu de deux, on peut envisager d'en faire un genre ou un sous-genre différent; dans ce cas, comme l'indique REICHENOW, 1953 (60), ce n'est pas le nom Nuttallia FRANÇA, 1909, qu'il faut employer, mais Achromaticus Dionısı), 1899, qui aurait priorité. L'espèce en question deviendrait donc soit Achromaticus equi (LAVERAN, 1901). soit Babesia (Achromaticu's) equ (LAVERAN 1901).

C'est THIROUX (cıté par BUCK, 1940 (9)) qui en 1903, a trouvé la piroplasmose du cheval à Madagascar ; à cette époque on ne connalssait pas encore l'existence de deux espèces, mais plus tard THIROUX a fait savoir qu'il s'agissait de la grande espèce, B. caballi. Depuis 1903, aucun cas n'est signalé jusqu'en 1939, quand BUCK trouve, par inoculation de sang à des chevaux, $B$. caballi et $B$. equi sur des mulẹts importés d'Algérie (BUCK, 1940) (9).

La piroplasmose et. la nuttallıose du cheval sont encore mentionnées dans le Rapporí Annuel de 1942; en 1950 BUCK et RAMAMBAZAFY (17), signalent le premier cas naturel d'infection par B. equir sur un cheval, né à Madagascar.

Depuls 1950, aucun cas de babésiose équine n'est plus signalé dans le pays.

Quel pourralt être le vecteur à Madagascar? II n'existe dans le pays que trois espèces d'lxodidae susceptibles de parasiter les chevaux : B. microplus, $A$, variegatum et $R h$. songuineus. D'après les tables de NEITZ (1956) (45) la transmissıon des Babesiae équines n'a été sıgnalée que pour $R h$. sangumeus; aucune espèce de Boophilus, ni d'Amblyomma ne figure dans ces. tables. 


\section{$*^{*} *$ \\ ESPÈCES OVINE ET CAPRINE}

Babesıa ovis (BABES, 1892) a été trouvée, par splénectomie d'un mouton indigène en 1933 (BUCK (5)). La babésiellose clinique ovine est mentionnée dans les Rapports Annuels de 1935. 1937. 1938, 1942 et 1947. Depuis 1947, nı le parasite, ni la maladie n'ont été signalés.

Notre Confrère RAYNAUD a splénectomisé (Rapport Annuel, 1960) trois moutons indigènes : le seul parasite qu'il a vu sortir était Theilerı sergenti (WENYON, 1926). (Il nous semble, après des recherches bibliographiques, que c'est ce nom qui a priorité pour l'espèce apathogène des ovins et caprins, l'espèce pathogène étant Thelleria ovis LITTLEWOOD, 1914 (voir par exemple CURASSON, 1943 (22) et REICHENOW, 1953 (60)). Mais l'historique de la nomenclature est extrêmement confus.)

La même année (Rapport Annuel, 1960), RAYNAUD met en évidence sur un mouton Eperythrozoon ovis NEITZ, ALEXANDER ef DU TOIT, 1934, après I'inoculation, par voie intraveineuse, de broyat d'organes d'Amblyommo variegatum (II n'est pas précisé s'il 's'agit d'un mouton splénectomisé ou non).

Nous avons splénectomısé 4 moutons indigènes (Rapport Annuel, 1962), sans trouver d'hématozoaires, pendant des périodes d'observation, variant de 35 à 38 jours (Notre confrère RAYNAUD a, en sus des 3 moutons mentionnés dans le Rapport Annuel de 1960, splénectomisé plusieurs autres moutons; d'après ses notes, if semble que la plupart était ındemne d'hématozoaires).

En 1963, nous avons vu de rares Th. sergenti dans le sang d'un mouton de Tananarive, envoyé au Laboratoire.

Nous avons eu, la même année, l'occasion de splénectomiser 7 chèvres indigènes; les périodes d'observation ont varié de 32 à 68 jours, sauf dans un cas où l'animal maurrait de salmonellose le $17 \mathrm{e}$ jour. Nous n'avons vu que sur une des chèvres de très rares $T$ h. sergenti, le $25^{\mathrm{e}}$ ef le $29^{e}$ jour ; ces hématozoaires ne sont plus apparus pendant le reste de la période d'observation. de 67 jours dans ce cas.

En conclusion : existence prouvée de Babesio ovis, Therlerio sergent, et Eperythrozoon ovis. Les maladies naturelles par les deux derniers parasites n'ont jamals été observées; $B$. ovis semble peu important.

$$
* * *
$$

\section{ESPÈCE PORCINE}

Pas d'hématozoaire signalé jusqu'en 1962, où nous avons eu l'occasion d'abserver, dans le sang d'une jeune truie sacrifiée in extremis, de nombreux hématozoaıres, vraisemblablement Babesia perroncitol (CERRUTI, 1939) (Rapport Annuel, 1962).

Nous avons, par la sulte, splénectómisé 7 porcs indigènes, dans l'espoir d'assıster à la sortie de ce parasite, afin de pouvoir mieux l'étudier. Aucune Babesia n'est apparue dans le sang des animaux , pendant des périodes d'observation variant pour 4 ! d'entre eux de 64 'à 89 jours ; un porc est mort de péritonite le $21 \mathrm{e}$ jour après l'opération (sans doute par suite de celle-ci), les deux autres n'ont pu être observés que pendant 19 et 20 jours, et ont dû être utilisés dans un autre but.

Ce résultat ne saurait surprendré, les animaux étant jeunes et en provenance de Tananarive, où la tıque $R h$. sanguineus, qui nous paraît le vecteur le plus probable, semble absente. Nous espérons pouvorr splénectomiser des porcs de régions où cette tique est commune.

Par contre, nous avons vu apparaître dans le sang des 4 animaux qui ont pu être suivis pendant longtemps, des petites structures sur ou dans les glabules rouges, quelquefois accolées sur la périphérie de ceux-ci, en forme de cocci, ou de forme irrègulière, parfois en petits bâtonnets, plus rarement en petits anneaux. Ces structures nous faisaient surtout penser aux Hoemobartonellae décrites chez d'autres espèces animales; elles étaient tout à falt différentes des Eperythrozoon du porc, sauf les petits anneaux, qui, parfois, étaient plus clairs que les autres structures (qui étaient violettes) ; il est possible qu'il y aıt eu de très faubles accès d'Eperythrozoon porvum SPLITTER, 1950, mais nous ne le pensons pas, puisque ces anneaux sont toujours restés à un taux très bas, el étaient présents en même temps que les autres structures. A notre connaissance, aucune espèce d'Hoemobartonella n'a été décrite chez le porc. Des injectıons de néoarsphé- 
namine n'avaient pas d'influence sur les infestations. Les structures apparaissaient de 1 à 5 semaines après la splénectomie, et persistaient pendant toute la période d'observation, en nombre variable; à certaines époques, pratiquement toutes les hématies en portaient au moins une.

$$
*^{*} *
$$

\section{ESPÈCE CANINE}

Le seul hématozoaịre du chien connu à Madagascar est Babesia conis (PIANA et GALLI-VALERIE, 1895). L'on indique son existence dans les Rapports Annuels de 1934, 1935, 1945 et 1959. BUCK ef LAMBERTON 1946 (14) observent le parasite chez des chiens à Tananarive. L'existence de Rh. sanguineus à Madagascar était connue (dès le Rapport Annuel de 1930) ; mais il n'avait jamais été observé à Tananarive. Or, il y est apparu en 1943 (BUCK, 1948 (10)), et en 1946 les auteurs cités ont eu l'occasion de voir B. conis. On mentionne encore la piroplasmose canine, sans autre précision, dans le Rapport Annuel de 1959.

Depuis, ce parasite n'a plus été diagnostiqué et, fait curleux, la tique $R h$. sanguineus semble brusquement avoir disparu de Tananarive (vorr plus loin) bien qu'elle soit encore commune sur la côte ; nous avons pu le constater en 1962 dans la région de Majunga, et plusieurs envols de cette espèce sont parvenus au Laboratare en 1961. 1962 et 1963, en provenance de différents endroits de la côte malgache.

Notre confrère, le Dr LAPEIRE, a eu l'occasion de pratiquer, en 1963, une splénectomie sur un chien de Tananarive, pour des raisons thérapeutiques. Le propriétaire n'a pas signalé de maladie à la suite de l'opération et le chien est toujours en vie, depuis plus d'un an. Nous avons récemment enlevé la rate d'un autre chien de Tananarive; rien n'a été à signaler dans les frottis de sang pendant une période d'observatıon de 50 jours. L'animal a été inoculé, le 15 e jour après l'opération, avec le broyat de la rate d'un chien, mort suspect de piroplasmose, ayant vécu dans des régions de la côte où $R h$. songunneus est fréquent. La rate avait été prélevée tout de suite après la mort de l'animal. Jusqu'à présent, un mois et demi après l'inoculation, aucun parasite n'est apparu dans le sang du chien d'expérience.

\section{*** \\ OISEAUX}

Aucun parasite sanguin n'a jamais été trouvé à Madagascar chez la poule, bien que la tique Argos persicus y ait été signalée.

Hoemoproteus columbae KRUSE, 1890 est commun sur les pigeons domestiques. Ce protozoaire a été trouvé pour la première fois en 1961 (Rapport Annuel, 1961). It est probable que la plupart des pigeons en sont porteurs, le vecteur Pseudolynchia canoriensis étant commun. Nous avons l'impression que cet hématozoaire peut parfois être pathogène, lors des primo-infections ; nous avons vu de la mortalité dans un petit élevage de pigeons, associée à la présence de nombreux $H$. columbae sur les pigeons malades; la maladie avait débuté peu de temps après que le propriétaire ait permıs aux pigeons de quitter le pigeonnier et il remarquait alors, pour la première fois, la présence d'ectoparasites, qui se révélaient être $P$. conariensis.

Nous avons récemment observé des Hoemoproteus, qui nous semblent appartenir à une autre espèce, sur des pigeons domestiques. Les graıns de pigment dans les gamétocytes étaient beaucoup plus fins que sur $H$. columbae, et le noyau des érythrocytes infestés était déporté sur le côté, ou vers une extrémıté. II pourrait s'agir d'Haemoproteus sacharovi NOVY et MACNEAL, 1904, mais nous n'avons pas eu l'accasion de comparer le parasite à des spécimens ou des dessins de ce dernier.

\section{REMARQUES SUR LES TIQUES DES ANIMAUX DOMESTIQUES A MADAGASCAR}

Les espèces suivantes ont été signalées:

\section{ARGASIDAE :}

Argos persicus (Oken, 1818).

Ornithodorus moubata (MURRAY, 1877).

Ornithodorus porcinus domesticus "WALTON, 1962.

Otoblus megnini (DUGĖS, 1883).

IXODIDAE :

Amblyomma variegatum (FABRICIUS, 1794).

Boophilus microplus (CANESTRINI, 1887).

Rhipicephalus sanguineus (LATREILLE, 1806). 
Périodiquement, il $y a_{1}$ avec des animaux importés, des introductions d'autres espèces d'lxadidae, dont aucune ne semble s'être maintenue dans le pays (voir plus loin).

II y a plusieurs autres espèces de tiques, trouvées sur des animaux sauvages. Nous n'en parlerons pas dans ce texte.

\section{Argas persicus :}

Cette espèce est signalée pour la première fois à Madagascar en 1932 (Rapport Annuel), trouvée à Majunga. BUCK, 1935 (7) la mentionne comme existante à Majunga. La collection du Laboratoire possède des spécımens marqués : Poule, Vohémar, 1936, sans autre précision. Son existence à Diégo-Suarez et Vohémar est signalée en 1946 (Rapport Annuel). BUCK (1948) (10) et le Rapport Annuel de 1948 mentionnent son existence dans les zones côtières, particulièrement dans le Nord. Depuis, cette tique n'a plus été trauvée. Nous l'avons cherchée en 1962 dans quelques poulaillers de la région de Majunga et la région d'Ambatondrazaka, sans résultat. Le Service Provincial de l'Elevage à Diégo-Suarez a examiné, sur notre demande, des poulaillers à Vohémar, en 1962, sans trouver la tique.

Elle existe dans les îles Maurice et de la Réunion (voir HOOGSTRAAL, 1953) (33).

Des maladies transmises par A. persicus, ni la spirochétose avıaire, ni l'aegyptianellose, n'ont jamais été signalées dans le pays.

\section{Ornithodorus moubato ef Ornithodorus porcinus :}

O. moubata a été ıdentıfié pour la première fois par CHATTON ef ROUBAUD (LAMOUREUX, 1913 (37)).

WALTON, 1962 (77) divise 0 . moubato en quatre espèces différentes; nous avons montré que les quelques tiques malgaches que nous avons pu examiner (UILENBERG, 1963) (76), appartiennent à l'espèce Ortnithodorus porcinus W'ALTON 1962 et se rapprochent le plus de la sous-espèce $O$. porcinus domesticus. II est possible que d'autres espèces du groupe moubata existent à Madagascar, mais nous n'avons pas eu l'occasion d'étudier d'autres exemplaıres.

La tique semble limitée à l'Ouest et au Centre du Pays (POISSON, 1931 (53), UILENBERG 1963 (76)), et y est fréquente dans les porcheries.
Elle ne semble pas jover de rôle important dans la pathologie des animaux domestiques.

\section{Otobius megnini :}

BUCK, 1948 (11) signale cette espèce pour la première fois à Madagascar. Il émet l'hypothèse de son introduction par avion d'Afrique, puisqu'il ne la trouve que dans les environs de Tananarive (BUCK, 1948 (11)) et puisqu'elle n'a pas été observée à Tamatave, où les animaux importés d'Afrique du Sud subissent une quarantaine. Mais le Rapport Annuel de 1949 la signale à Tamatave, sur un bovin importé d'Afrique du Sud ; il nous semble donc plus probable qu'O. megnin ait été introduit avec du bétall en provenance d'Afrique du Sud où il est commun (THEILER et SALISBURY, 1958 (71)), et transporté dans la région de Tananarive à partir de Tamatave. II n'est pas étonnant que cette tique n'ait pu être trouvée à Tamatave, autrement que sur un bovin ımparté, puisque le climat de Tamatave est très humide, et $O$. megnini ne se maintient pas dans ces conditions (THEILER et SALISBURY, 1958 (71)). En 1949, I'on signale également son. existence dans la région de Betroka (Sud du. pays).

Actuellement, il est très commun, à longueur d'année, dans la région de Tananarive, ef n'est pas signalé allleurs (Mais il échappe facılement à l'attention lors d'un examen superficiel).

Les chevaux de la Gendarmerie à Tananarive sont très parasités; la présence des tiques dans les oreilles les irrite et Il est quelquefois difficile, d'après les gendarmes, de travailler avec ces chevaux. Les autres anımaux, en particulıer les bovins, ne semblent pas souffrir de façon sensible de sa présence dans les oreilles, et la transmission de maladies n'a, à notre connaissance, pas été signalée.

\section{Amblyomma variegatum :}

La tique est présente à Madagascar depuis longtemps. POISSON, 1927 (51) la signale. Elle est très répandue, surtout dans les régions assez basses et chaudes; elle est beaucoup moins commune sur les Hauts-Plateaux. On la rencontre pourtant dans la région de Tananarive, mass en petit nombre: le climat ne semble pas lur convenir. Les bceufs conduits à pied à l'abattoir 
de Tananarive, surtout en provenance de l'Ouest du Pays, en apportent continuellement, et se révèlent, examinés à l'abattoir, très souvent infestés; les adultes dominent pendant la saison chaude et humide, les nymphes pendant la saison sèche (comme l'a signalé HOOGSTRAAL, 1956 (34) pour d'autres pays). Les bovins nés dans la région tananarivienne en sont beaucoup moins souvent porteurs, et nous ne savons pas si la population d'A. variegatum se maintiendrait sur les plateaux sans l'apport continuel par les boufs de l'Ouest.

C'est une espèce redoutable, parce qu'elle transmet la heart-water à Madagascar, maladie Importante, et qui le deviendra encore plus avec l'extension de bovins de race importée ou améliorée. BUCK, 1935 (7) et 1948 (10) l'accuse également de jouer un rôle dans la propagation de la streptothricase cutanée des bovins et de la lymphangite ulcéreuse des équidés.

\section{Boophilus microplus :}

Cette espèce semble ubiquitaire dans le pays ; elle est aussi commune sur les plateaux que dans les zones côtières.

Elle est déjà mentionnée dans le premier Ropport Annuel 1929 (sous le nom de Margaropus onnulatus) et avait d'ailleurs été Identifiée en 1901 par NEUMANN (cité par MINNING, 1934 (43)) comme B. decolarotus. L'historıque de son identification et de sa nomenclature à Madagascar a été très confus (UILENBERG, 1962 (75) synonymie et bibliographie).

B. microplus est influencé, dans une certaine mesure, par les saisons, mais l'on en trouve tous les stades à longueur d'année, tout au moins sur les plateaux.

Son importance tient dans le fait qu'il transmet divers hématozoaires importants; les effets nuisibles directs sont également considérables (voir LITTLE, 1963 (40)).

\section{Rhipicephalus sanguineus :}

L'espèce est déjà mentionnée dans le Rapport Annuel de 1930. II n'a pas été fait d'études morphologiques ef biologiques sur $R h$. sanguineus à Madagascar ; aussi nous ne pouvons affirmer s'il s'agit de Rh. sanguineus sensu stricto et de quelle race biologique (voir MOREL et VASSI-
LIADES, 1962 (44)) ; le chien est de toute façon I'hôte normal à Madogascar, et. nous n'avons pas trouvé de référence sur son parasitisme chez d'autres animaux.

Rh. sangumeus ne semble actuellement plus exister dans la région tananarivienne; le Dr BUCK a remarqué sa disparition, depuis quelques années, de la ville (communication personnelle), et nous ne l'y avons jamais trouvé. Ce fait est inexpliquable pour nous, puisqu'il y était apparu en 1943 (BUCK, $1948(10)$ ), et même en nombre important (communication personnelle du Dr BUCK). Or, le climat de Tananarive semble convenir parfaitement à l'évolution de la tıque. Certains attribuent la disparition de $R h$ i. sanguineus de Tananarive aux pulvérisations d'insecticides contre les anophèles, dans la lutte anti-paludique; ceci nous paraît invraisemblable, étant donné le grand nombre de chiens errants dans la ville, dont beaucoup ne rentrent jamais dans une maison.

$R h$. sanguineus doit être incriminé comme le vecteur de la babésiose canine à Madagascar.

Nous renvoyons également à quelques remarques sur cette espèce que nous avons' faites plus haut, à propos de Babesia canis.

Nous mentionnerons d'autres espèces de tıques trouvées uniquement sur des anımaux importés, dans la liste des arthropodes ci-dessous. Aucune de ces espèces 'ne semble s'être établie dans le pays.

\section{LISTE DES PROTOZOAIRES, RICKETTSIALES, ET ARTHROPODES DES ANIMAUX DOMES- TIQUES A MADAGASCAR}

Cette liste donne tous les protozodires et Rickettsiales déterminés jusqu'ici dans le pays, pour autant que nous ayons pu nous en assurer (et en nous basant surtout sur les Rapports Annuels du Laboratoire). En ce qui concerne les arthropodes: nous ne mentionnons pas la plupart des insectes ailés, tels imoustiques, mouches etc..., dont la liste seralt trop longue et qui ne sont pas des ectoparasites spécifiques.

Nous indiquons après le nom de chaque parasite, l'année où celui-ci fut signalé à Madagascar pour la première fois (pour autant que nous ayons pu trouver l'année), sans autre mention s'il s'agit d'un. Rapport Annuel, avec le nom de l'auteur s'il s'agitld'une autre publication. Les 
hôtes, sur lesquels l'espèce en question a été trouvée à Madagascar, sont également indiqués. Les parasites, dont le nom est placé entre parenthèses, n'ont pas, dans notre opinion, été déterminés de façon certaine.

\section{Protozoa}

Babesio argentino (LIGNIERES, 1909). Bovin, (BUCK, 1937 (8)).

B. bigemina (SMITH ef KILBORNE, 1893). Bovin, buffle domestique (CAROUGEAU, 1906, voir BUCK, 1934 (6)).

B. caballi (NUTTALL, 1910). Equidés (THIROUX, 1903, voIr BUCK, 1940 (9)).

B. conis (PIANA et GALLI-VALERIE, 1895). Chien (1934).

B. equi (LAVERAN, 1901). Equidés (BUCK, 1940 (9) sur mulet importé, BUCK et RAMANBAZAFY, 1950 (17) premier cas naturel, sur cheval).

B. ovIs (BABES, 1892. Mouton (BUCK, 1933 (5)).

(B. ? perroncitol (CERRUTI, 1939)). Porc (1962):

Balantidium coli (MALMSTEN, 1857). Pore (1960; c'est la première année où cette espèce est mentionnée dans le Rapport Annuel, mas elle était vraisemblablement connue auparavant, tout au morns chez l'homme).

Eimerio perforans LEUCKART, 1873. Lapin (1962).

E. stiedae (LINDEMANN, 1865). Lapin (1931).

E. tenella (RAILLIET et LUCET, 1891). Poule (1962; la coccidiose des volailles est connue depuis longtemps, mais aucune espèce n'avait été déterminée).

E. zürnı (RIVOLTA, 1878). Bovin, (1962); sa présence avait déjà été soupçonnée en 1937).

Encephalitozoon cuniculi LEVADITI, NICOLAU et SCHOEN, 1923. Souris blanche (n'a pas encore été observé à Madagascar chez des animaux domestiques) (SUREAU, 1963) (65).

Haemoproteus columbae KRUSE, 1890. Pigeon domestique (1961).

(H. ? sachorow NOVY ef MACNEAL, 1904). Pigeon domestique (Observé en 1964).

Isosporo felis WENYON, 1923, Chat (1957)

Leishmania donovon (LAVERAN ef MESNIL. 1903). Chien importé depuis un an de la région méditerranéenne (BUCK e. a. 1951 (12)) (il ne semble pas avoir été trouvé à Madagascar de
Phlebotomus qui pique les animaux à sang chaud :

BUCK e. a., 1952 (13)).

Sarcocystis fusiformis RAILLIET, 1897. Bovin (1951).

Theileria mutans (THEILER, 1906). Bovin (1932).

Th. sergenti (WENYON, 1926). Mouton, chèvre (1960).

Toxoplasmo gond" NICOLLE et MANCEAUX, 1908. Lemur cotto (SUREAU e. a., 1962 (66)) plus tard trouvé chez un animal domestique, le pigeon (SUREAU et UILENBERG, $1963(6 \mathrm{~T})$ ).

Trichomonas fotus RIEDMÜLLER, 1928. BovIn (BUCK et QUESNEL, 1950 (16)).

T. gallınae (RIVOLTA, 1878). Pigeon domestıque (1961).

Tryponosomo thesleri LAVERAN, 1902, Bovin (1961).

La liste des protozoaires des animaux domestiques malgaches est certainement très loin d'être complète. Aucune étude systématique n'a été farte des coccidies par exemple, bien que les coccidioses soient importantes dans le pays. Rien n'est connu sur les protozoaires' apathogènes du tube digestif.

\section{Rickettsiales}

Anaplasma marginale THEILER, 1910. Bovin, buffle domestique (CAROUGEAU, 1913 (19)).

A. centrale THEILER, 1911. Bovin (souches importées en 1960 et 1963, et répandues par la prémunition artificielle).

(? Colesiota conjunctivae (COLES, 1931)). Mouton (1940).

Coxiello burnet" (DERRICK, 1939). Homme (PORTE e. a. 1959 (54) ; il s'agit de diagnostics sérologıques; jusqu'à maıntenant la fièvre $Q$ n'a pas été diagnostiquée sur des anımaux à Madagascar).

Cowdria ruminantrum (COWDRY, 1925). Bovin, mouton, chèvre. (La heartwater fut diagnostiquée à Madagascar pour la première fois en 1925 : (ALEXANDER, 1931. (1)).

Eperythrozoon ovis NEITZ, ALEXANDER et DU TOIT, 1934. Mouton (1960).

E. tegonodes HOYTE, 1962. Bovin (Reconnu à Madagascar depuis 1963).

E. wenyoni ADLER et ELLENBOGEN, 1934. Bovin (1960). 


\section{Arthropoda}

\section{Diptera :}

(Gasterophilus ? intestinahs (DE GEER, 1776)) (1929, sous le nom Oestrus equi, sans précision de l'hôte).

Hypodermo lineotum (DE VILLERS, 1789). Bovins Importés de la France (observée en 1962 à Tananarive; l'hypodermose des bovins, sans autre précision, a été signalée dans le Rapport Annuel de 1935. Il semble qu'aucune espèce d'Hypoderma ne se solt établie à Madagascar). Melophagus ovinus (LINNÉ, 1758). Mouton (1948).

Oestrus ovis LINNE, 1761. Mouton, chèvre (1929).

Pseudolynchia conoriensis (MACQUART, 1840). Pigeon domestique (1931).

\section{Phthiraptera :}

Colpocepholum turbinatum DENNY,1842. Pigeon domestıque (déterminé en 1963).

Columbicola columbae (LINNÉ, 1758). Pigeon domestique (1961).

Domalinio bovis (LINNÉ, 1758). Bovin (1934).

D. equi (DENNY, 1842). Ane (1948).

D. ovis (SCHRANK, 1781). Mouton (1959).

Damalinia sp. Chèvre (déterminée en 1963).

Gliricolo porcelli (SCHRANK, 1781). Cobaye (1962).

Goniocotes gollinae (DE GEER, 1778). Poule (1955).

Goniocotes sp. Pigeon domestique (1949).

Gyropus ovalis BURMEISTER, 1838. Cabaye (1962).

Haemotopinus eurysternus (NITZSCH,1818). Bovin (1929).

(H. palpebrae GRETILLAT, 1957). Bovin (GRETILLAT, 1957 (28)) ; nous n'avons pas pu observer de différence avec $H$. eurysternus et considérons, avec le Dr CLAY du Britısh Museum (correspondance $H$. polpebroe comme synonyme d'H. eurysternus.

H. suis (LINNÉ, 1758). Porc (1947).

Holomenopon sp. Canard domestique (Déterminé en 1963).

Linognathus africonus KELLOGG et PAINE, 1911. Mouton, chèvre (1956).

L. stenopsis (BURMESITER, 1838). Chèvre(1946).
L. setosus (OLFERS, 1816). Chien (1956).

L. vifuli (LINNÉ, 1758). Bovin (1929).

Lipeurus caponis (LINNÉ, '1758). Poule (1955).

Menaconthus cornutus (SCHOMMER, 1913). Poule (Déterminé en 1963).

Menaconthus sp, Canard domestıque (Déterminé en 1963).

Menopon gallinoe (LINNÉ, 1758). Poule (1929).

Trichodectes canis' (DE GEER, 1778). Chien (1948).

\section{Siphonaptera.}

(Nous avons puisé largement dans le livre de LUMARET (1962) (41); nous ne connaissons pas l'année dans laquelle les espèces ont été signalées à Madagascar pour la première fois. Nous ne parlons que des espèces qui ont été trouvées sur des animaux domestiques malgaches.)

Ctenocephalides canis (CURTIS, 1826). Chien, chat, homme (? mouton) (voir remarques cidessous).

C. felis felis (BOUCHE, 1835). Chien (observé en 1964).

C. felis strongylus (JORDAN, 1925), Chien, chat, mouton, chèvre, homme.

Echidnophaga gallinaceo (WESTWOOD, 1875). Poule, chien, homme.

Pulex irritans LINNÉ, 1758. Animaux domestiques (LUMARET, sans précisions), 'homme.

Tungo penetrans (LINNÉ, 1758). Pórc, chien (? bœuf), homme.

Ctenocepholides felis strongylus est l'espèce prédominante de ce genre à Madagascar. Nous n'avons trouvé qu'une seule fois une puce qui correspondait morphologiquement à $C$. felis felis. Nous n'avons jamais observé $C$. canis, et nous pensons que l'infestation massive par $C$. canis, signalée dans le Rapport Annuel de 1939, sur des moutons, a plutôt été par $C$. felis strongylus, le diagnostic ayant souvent été fait dans le temps d'après le seul profil de la tête (voir LUMARET, 1962 (41)). Nous avons pu voir en 1963 de massives infestations par $C$. felis strongylus sur des moutons et des chèvres.

\section{Hemiptera :}

CImex hemipterus FABRICIUS 1803. Habitation (n'a pas, à notre connaissance, été sighalé 
comme parasite des animaux à Madagascar) (Nous ne connaissons pas l'année où ce parasite fut signalé pour la première fois dans le pays ; GRUCHET (1961) (30) le mentionne).

Triatoma rubrofosciata (DE GEER, 1773) (n'a pas encore, à notre connaissance, été signalé comme parasite des anımaux à Madagascar) LE GAC, 1937 (78).

\section{Ixodoidea :}

Amblyomma hebraeum $\mathrm{KOCH}$, 1844. Bovin, mouton (trouvé uniquement sur des animaux importés d'Afrıque du Sud, à Tamatave) (Rapports Annuels de 1945, 1946 et 1949 ; la collection du Laboratoire possède des exemplaires datant de 1944).

Amblyomma variegotum (FABRICIUS, 1794). Bovin, mouton, chèvre, cheval, porc, chien, poule, pintade, Pterocles personatus, homme ; HOOGSTRAAL (1953) (33) le signale cussi sur : Centropus toulou toulou. Tenrec ecaudatus et Chomaeleo pardalis (POISSON, 1927 (51)).

Argas persicus (OKEN, 1818). Poule, poulaller (1932).

Boophilus microplus (CANESTRINI, 1887). Bovın, mouton, chèvre, daim, cheval, chien, hamme (NEUMANN, 1901, cité par MINNING, 1934(43)).

Dermacentor rhinocerınus (DENNY, 1843) Bovin (trouvé sur un bovin importé du Kenya à Tamatave) (1961).

Hyalomma marginatum rupifes $\mathrm{KOCH}, 1844$ (? Bovin) mouton, chèvre (trouvé uniquement sur des animaux importés d'Afrique du Sud, à Tamatave). (HOOGSTRAAL, 1953 (33) et THElLER, 1956 (70) ; la collection du Laboratare en possède des exemplaires marqués: moutons importés d'Afrique du Sud, sans précision de l'année. Les Rapports Annuels de 1946 et 1949 mentionnent $H$. impressum, sur chèvre et bovins importés d'Afrique du Sud ; I'identification ayant été faite à Onderstepoort, ce nom peut aussi bien s'appliquer à $H$. marginatum rupıfes qu'à $H$. truncotum, pulsque ces espèces étaient appelées à cette époque en Afrıque du Sud respectivement $H$. impressum rupifes et $H$. impressum transiens (vorr THEILER 1943 (69) et 1956) (70)). L'espèce $H$. impressum Koch, 1844 est, de toute façon, absente d'Afrique du Sud, d'après Hoogstraal 1956 (34) et THEILER, 1956 (70)).

H. truncotum $\mathrm{KOCH}, 1844$ (? Bovin) chèvre
(Trouvé sur des anımaux importés d'Afrique du Sud d̀ Tamatave, et en 1963 sur des chèvres importées d'Afrique du Sud, à Ambovombe ; voir plus loin) (HOOGSTRAAL, 1953 (33), THEILER, $1956(70)$ et observation en 1963. Voir remarques concernant $H$. impressum ci-dessus).

Morgaropus winthem! KARSCH, 1879. Bovin (Trouvé uniquement à Tamatave, sur bovins importés d'Afrique du Sud) (Rapport Annuel de 1946 et le Laboratoire possède 'des spécimens marqués : bovins importés d'Afrıque du Sud, Tamatave, 1945).

(Ornithodorus ? moubato (MURRAY, 1877)) Porcherie, habitation (LAMOUREUX, 1913 (37)). L'existence à Madagascar d'O. moubata sensu Walton n'a pas été prouvée).

0 . porcinus domesticus WALTON, 1962. Porcherie (UILENBERG, 1963 (76)).

Otoblus megnini (DUGĖS, 1883). Bovin, mouton, cheval, chien (? homme ; il s'agit d'une communication personnelle d'un gendarme, qui affirmait avoir eu cette tique dans une oreille) (BUCK, 1948 (11)).

Rhipicepholus appendiculotus NEUMANN, 1901. Bovin (importé d'Afrique du Sud, à Tamatave) (1949).

Rh. evertsi NEUMANN, 1897. Bovin, mouton, chèvre (Sur animaux importés d'Afrıque du Sud à Tamatave, et en 1963 sur chèvres importées d'Afrique du Sud, d̀ Ambovombe; vorr plus loin) (1944, 1945,1946, 1949 et observation en 1963).

Rh. sanguineus (Latreille, 1806). Chien (1930). (?Rh. simus KOCH, 1844) (Signalé à Madagascar par NEUMANN (1901) cité par HOOGSTRAAL, 1956 (34), 'et par POISSON (1927) (51), qui se base sur le Professeur BRUMPT. Nous n'avons jamals rencontré cette tique dans le pays et aucun auteur local n'y fait d'allusion directe. Nous ne croyons pas que $R h$. simus existe à Madagascar. Voir HOOGSTRAAL, 1953 (33)).

Le fal que Madagascar est une île, l'a protégé contre l'introduction de la plupart des espèces de tıques africaınes. La liste des tiques des animaux domestiques, établie dans le pays, est courte. Le danger que constituent les animaux importés, ne doit pas être sous-estimé. Aınsi Otobıus megnıni a-t-il été introduit récemment. Des chèvres mohair ont été importées d'Afrique du Sud en 1963 ; elles n'ont pas été mises en quarantaine 
à Tamatave, puisque le climat y est trop humide pour ces animaux; elles ont été transportées à Ambovombe (Sud du pays), régıon sèche, qui semble parfaitement convenir à l'évolution de Rhipicepholus evertsı et Hyalomma truncotum, espèces trouvées sur les chèvres après leur arrivée à Ambovombe. Nous ne savons pas encore si ces tiques ont réussi à s'y établir.

\section{Autres Acarina :}

Bdellonyssus bursa (BERLESE, 1888). Poule, homme (GRETILLAT, 1956 (27)).

Cnemidocoptes mutans (ROBIN et LANQUETIN, 1859). Poule (1929).

Cytodites nudus (VIZIOLI, 1870). Poule (1943).

Demodex bovIs STILES, 1892, BovIn (GEOFFROY, 1907, cité par POISSON, 1930 (52)).

D. conis LEYDIG, 1859. Chien (1930).

D. phylloides Csokor, 1879. Porc (1930).

(? Dermanyssus gallinae (DE GEER, 1778)). Poule (1929, nous n'avons jamais rencontré ce parasite à Madagoscar : des exemplaires dans la collection du Laboratoire, identifiés comme D. gallinae (1940 et 1953), se révélaient être Bdellonyssus bursa).

Laminosioptes cysticola (VIZIOLI, 1870). Poule (1951).
Notoedres cati (HERING, 1838). Chiat (1931).

Psoroptes equi bovis (GERLACH, 1857). Bovin (POISSON, 1930 (52)).

P. equi cunculi (DELAFOND, 1859), Lapin (1930)

P. equi ovis (HERING, 1838). Mouton (1934).

Sarcoptes scabie! (LINNE, 1758). Bovin, cheval, porc (1933).

Tyroglyphus sp. Poule (GRETILLAT, 1956.(27)).

Tyrophagus putrescentioe (SCHRANK, 1781). Buffle (GRETILLAT, 1958 (29) ; eu égard à la différence entre la description du liquide des kystes pulmonarres 'dans le rapport d'autopsie, et la description du contenu des kystes à l'arrivée au Laboratoire, nous penson's qu'il a pu s'agır d'acariens qui se sont développés dans le liquide après la mort de l'animal, plutôt que de vrais parasites ; voir GRETILLAT, 1958 (29)).

Nous pensons avair rassemblé dans ces listes pratiquement tous les parasites (des groupes indiqués) des animaux domestiques, identifiés dans le pays. L'on voit qu.il y reste beaucoup à rechercher.

\author{
Laboratorre Central \\ de l'Elevage Tananarive \\ Service d'Entomo-Protozoologie.
}

\section{SUMMARY}

A note on protozoas and ticks of domestic animals in Madagascar

Research carried out at Madagascar on protozoans and Rickettsiales of the blood of domestic anımais is described.

A short discussion points out why Babesio argentina in considered the valid name for the small bovine species in Madagascar.

Practically all aduit bovines living in an environment infested by ticks are carriers of $B$ bigemina ; $B$. argentina is much less frequent. The majority of bovines treated regularly for ticks do not carry Bobesid; it is above all in these animals that clinical babesiasis is seen Babesielliasis is much more common than true piroplasmasis partly perhaps because $B$. orgentino is rare and so natural premunition is not common. The cerebral form of bobesieliosis is common while that of true piroplasmosis has not been seen. Acriflavin (Gonacrin) and quinuronium sulphate (Zothelone) are effective for both; pentamidine (Lomidine) is only effective against true piroplasmosis. Premunition agaınsi babesiellasis presents a problem since $B$. argentina is not regularly transmitted with the blood of chronic carriers. Calves of less than one month are remarkably resistant to infection by $B$. bigemino; a clinical reaction to $B$. argentina is more common. The duration of the state of premunition seems often to be quite shart (less than a year). 
Bovine anaplasmosis is noi common. There are scarcely any adult cattle in tick infested areas who are not carriers of A. marginale. A large proportion of cattle regularly treated for ticks, the majority of whom do not carry Babesia, are nevertheless infected with $A$. marginale. Terramycin is satisfactory for the treatment of anaplasmosis.

Eperythrozoon wenyoni and E. teganodes are very widespread. They do not appear to follow any rule. The two species have appeared in splenectomized calves, sheltered from ticks, and having never shown them during the 5 to 8 months following splenectomy. The means of 'transmission remains unknown. The two species may be pathogenic even for bovınes not splenectomized, but no deaths have been noled. Neoarsphenamine is effective.

A brief expose is given on the three species of Argasidae and the species of Ixodidae of domestic animals in Madagascar. Several other species of tick have been introduced with imported cattle, but do nol appear to have established themselves in the country.

A list of protozoans, Rickeltsiales and arthropods, parasitic on domestic animals in Madagascar, is given.

\section{RESUMEN}

Observación en los hematozoos e ixodos de los animales domésticos en Madagascar.

Se notan las investigaciones hechas en Madagascar en los hematozoos y las rickettsias de la sangre de los animales domésticos.

Una breve discusión muestra par qué Babesia argentina (Lignières, 1909) está considerada como no valida en la pequena especie bovina de Madagascar.

Casi todos los bovinos adultos, viviendo en un medio ambiente infectado con ixodos, albergan la B. bigemina ; es mucho más escasa la B. argentina, no albergan Babesia la mayor parte de los bovinos regularmente deparasitados, y es sobretodo en estos animales que son observadas las Babesiolosis clinicas. Es mucho más frecuente la Babesiolosis que la verdadera Piroplasmosis, tal vez en parte por que $B$. argentina es rara y la premunición natural poco frecuente. A menudo se encuentra la forma cerebral de la Babesiolosis, sino la de la verdadera Piroplasmosis. La acriflavina (Gonacrina) y el quinuronium sulfato (Zotélonio) actuan en las dos enfermedades ; es eficaz la pentamidina (Lomıdina) sálo contra la verdadera Piroplasmosis. La premúnición contra la babesiolosis establece problema por qué no está transmitida 'regularmente $B$. argentina con la sangre de los portadores crónicos. Los lerneros de menos de un mes son nolablemente resistentes contra la infección por $B$. bigemino; es más frecuente una reacción clinica con B. argentina. La duración del estado de premunición parece a menudo ser demasiado breve (menos de un ano).

No es frecuente la Anaplasmosis bovina. En un medio ambiente no infectado con ixodos, hay pocos bovinos aduitos no portadores de A. morginale. Un termino importante de los bovinos regularmente deparasıtados, y cuya mayor parte no alberga Babesia, estan infectados sın embargo por A. morginale. Luego parece que otros vectores que los ixados podrian desempenar un papel. Son muy resistentes contra la infección de $A$. marginale los terneros de menos de un mes. La Terramicina da satisfacción en el tratamiento de la Anaplasmosis. 


\section{BIBLIOGRAPHIE}

1. ALEXANDER (R. A.). - Heartwater. The present state of our knowledge of the disease. 17 th Report Director Vet. Serv. \& An. Ind., South Africa, 1931, part I : 89-150.

2. ANGELOVSKI (T.). - (Nous n'avons lu qu'un abstrait dans :) Vet. Bull., 1958, 28 : 297.

3. BABES $(V$.$) .- Sur l'hémoglobinurie bacté-$ rienne du bœuf. $C$. R. Séances Acad. Sciences, Parıs, 1888, Tome 107:692.

4. BABES (V.). - Die Aetiologie der seuchenhaften Hamoglobinurie des Rindes. Arch. f. pathol. Anatomie u. Physiologie u. f. klinische Medicin, 1889, 115 (Folge 11, Band 5) : 81-108.

5. BUCK (G.). - Existence de Babesiella ovis à Madagascar. Bull. Soc. Path. exot., 1933, $26: 1127$.

6. BUCK (G.). - Les piroplasmoses des bovidés à Madagascar. Bull. Economique du Gouvernement Général de Madagascar et Dépendances, 1934 (96, novembre) : 978981.

7. BUCK (G.). - Les tiques à Madagascar ef les maladies qu'elles inoculent aux animaux domestiques de la Grande lle. Revue Agricole de Mourice, 1935 (84) : 196-209.

8. BUCK (G.) - - Existence de Babesiella berbera à Madagascar. Bull. Soc. Path. exot., 1937. $30: 436-437$.

9. BUCK (G.). - A propos des piroplasmoses des équidés à Madagascar. Bull. Soc. Path. exot., 1940, 33 : 86-89.

10. BUCK (G.). - Tiques des animaux domestiques à Madagascar. Bull. Agricole Madagascar et Dépendances, 1948, 1 (4) : 3-11.

11. BUCK (G.). - - Existence d'Ornithodorus, megnini Dugès à Madagascar. Bull. Soc. Path. exot., 1948, $41:$ 567-568.

12. $\operatorname{BUCK}(G),. C O U R D U R I E R$ (J.), DOREL (R.) et QUESNEL (J. J.). - Premier cas de leishmaniose canine à Madagascar. Bull. Soc. Path. exot., 1951, $44:$ 428-430.

13. BUCK (G.), COURDURIER (J.) et QUESNEL (J. J.). - A propos de Phlebotomus squamipleuris. Communication à la Soc. Sci. Méd. Madagascar, le 4 février 1952.
14. BUCK (G.) et LAMBERTON. - La piroplasmose canine à Madagascar. Bull. Soc. Path. exot., 1946, $39: 283$.

15. BUCK (G.) et METZGER. - Note sur la babésiellose à Babesiella berbera chez des zébus, des métis-limousins et des limousins purs à Madagascar. Bull. Soc. Path. exot., 1940, $33: 89-93$.

16. BUCK (G.) et QUESNEL. — Premières observations de trichomonoses bovines à Madagascar. Bulf. Soc. Path. exot., 1950, 43 : 521523.

17. BUCK (G.) et RAMAMBAZAFY, - Premier cas de nuttaliose naturelle signalée à Madagascar. Bull. Soc. Path. exot., 1950, $43: 243$.

18. CALLOW (L. L.) et HOYTE (H. M. D.). Transmission experiments using Babesia bigemina, Theileria mutons, Borrelia sp. and the cattle tick. Boophilus microplus. Aust. Vet. J., 1961, $37: 381-390$.

19. CAROUGEAU. - Des maladies dans l'acelimatation d'animaux importés. Existence de l'anaplasmose à Madagascar. Bull. Soc. Sci. Méd. Madagascar, 1913, $7:$ 31-35.

20. CERNAIANU (C. C.). - Piroplasme si piroplasmoze. 1958. Tome II. Partea speciala. Editura Academiei Republicii Populare Romîne.

21. CORNIL (A. V.) ef BABES (V.). - Hemoglobinurie bactérienne du bœuf. Les Bactéries. 1890. 3e Edition, Tome I, Félix Alcan Editeurs, Paris : 350-354.

22. CURASSON (G.). - Traité de Protozoologie vétérinaire et comparée. 1943. Tome III, Sporozoaires. Vigot Frères Editeurs, Paris.

23. DAVIES (S. F. M.), JOYNER (L. P.) ef KENDALL (S. B.). - Studies on Babesia divergens (M'Fadyean' and Stockman, 1911). Ann. Trop. Med. Paras., 1958, $52:$ 206-215.

24. DAVIES (S. F. M.), JOYNER (L. P.) ef KENDALL (S. B.). - Validity of the species Babesia divergens (M'Fadyean and Stockman, I9II). Trans. Roy. Soc, trop. Med. Hyg., 1958, 52 : 302.

25. FEIDER (Z.), RAUCHBACH (C.) et MIRONESCU'(I.). - Die Zecken der Rumànischen Volksrepublik. Ceskoslovenska parasitologie, 1958, $5: 71-87$. 
26. FEIDER (Z.), RAUCHBACH (C.) et MIRONESCU (I.). - Contributie la cunoasterea genului Hyolomma (Acari, Ixodoidea) in R. P. R. Academia R. P. R. Filiala lasi Studii sı Cercetari Stıntifice Bıologie si St. Agricole, 1958, $9: 31-40$.

27. GRÉTILLAT (S.). - Deux acariens parasites de Gallus domesticus Lin. à Madagascar. Rev. Elev. Méd. vét. Pays trop. 1956, 9, 359 365.

28. GRÉTILLAT (S.). - Haematopinus polpebrae n. sp. (Siphunculata) parasite du zébu à Madagascar. Ann. de Parasitologie, 1957, $32: 167-172$.

29. GRÉTILLAT (S.). - Kystes pulmonaires à acarlens chez une bufflesse. Bull. Soc. Path. exot., 1958, 51 : 536-539.

30. GRUCHET (H.). - Sensibilité de Cimex hemipterus Fabr. 1803, au DDT, à la Dieldrine ef aux mélanges DDT + Diazinon et Dieldrine + Diazinon dans la région de Miandrivazo, Madagascar. Bull. Soc. Path. exot., 1961, $54: 1358-1365$.

31. HALL (W. T. K.). - The immunity of calves to Babesia orgentina infection. Aust. Vet. J., 1960, $36: 361-366$.

32. HENNING (M. W.). - Animal diseases in South Africa. 1956. 3e Edition. Central News Agency Lid., South Africa.

33. HOOGSTRAAL $(\mathrm{H}$.$) . - Ticks (lxodoidea) of$ the Malagasy faunal region (excepting the Seychelles). Their origins and host-relationships ; with descriptions of five new Haemaphysalls species. Bull. Mus. Comp. Zool. Harv., 1953, 111 : 37-113.

34. HOOGSTRAAL (H.). - African Ixodoidea. 1956. Volume 1. Ticks of the Sudan. Research Report NM 005.050.29.07., Dept. of the Navy, Bureou of Medicine and Surgery.

35. HOYTE (H.M. D.). - Eperythrozoon teganodes sp. nov. (Rickettsiales) parasitic in cattle. Parasitology, 1962, 52 : 527-532.

36. KOTLAN (A.), VERSENYI (L.) et JA$\mathrm{NISCH}(M$.$) . - Uber das Vorkommen von$ Piroplasmo bigeminum in Ungarn. Acta Veterinaria Academıa Scientiarum Hungaricae, 1959, $9: 131-133$.
37. LAMOUREUX(A, ), - Présenced'Ornithodorus moubata dans un foyer de fièvre récurrente à la côte ouest de Madagascar. Bull. Soc. Path. exot., 1913, 6 : 146-149.

78. LE GAC (P.). - Note sur la présence d̀ Diego-Suarez (Madagascar) de Triatoma rubrofasciata (de Geer, 1773). Bull. Soc. Path. exot., 1937. (30) : 286-7.

38. LIGNIĖRES (J.). - La piroplasmose bovine. Nouvelles recherches ef observations sur la multipliclté des parasites, leur évolution, la transmission naturelle de la maladie ef la vaccination. Arch. de Parasitologie, 1903, $7: 398-407$.

39. LIGNIERES (J.). - La prophylaxie et la pathologie des maladies protozoaires (piroplasmoses, trypanosomes, etc.) avec démonstration des parasites spécifiques ef des, animaux transmetteurs (tiques, moustiques, etc.). 9 e Congrès International de Médecine Vétérinaire à la Haye, 1909, S. G. 7, $3: 1-18$.

40. LITTLE (D. A.). - The effect of cattle tick infestation on the growth rate of cattle. Aust. Vet. J., 1963, $39:$ 6-10.

41. LUMARET (R.). - Faune de Madagascar, XV. Insectes Siphonaptères. Institut de Recherche Scientıfique de Madagascar, ITananarive, 1962.

42. MAHONEY (D. F.). - Bovine babesioses : diagnosis of infection by a complement fixation test. Aust. Vet. J., 1962, 38 : 48-52.

43. MINNING (W.). - Beitràge zur Systematik und Morphologie der Zeckengattung Boophilus Curtice. Z. Parasitenk, 1934, 7 : 1-43.

44. MOREL (P. C.) et VASSILIADES (G.). Les Rhipicepholus du groupe sanguineus: espèces africaines (Acariens: Ixodoidea). Rev. Elev. Méd. vét. Pays trop., 1962, 15 : 343-386.

45. NEITZ(W.O.). - Classification transmission, and biology of piroplasms of domestic animals. Ann. N. Y. Acad. Sci., 1956, 64 : 56-111.

46. NEITZ (W. O.). - Rapport de la deuxième Réunion du Groupe d'Experts F. A. O./O.I.E. sur les maladies du bétail transmises par des tiques. Le Caire : 1962, 36-37. F. A. O., Rome. 
47. PAVLOV (P.). - La lutte contre les piroplasmoses dans les conditions actuelles et les résultats obtenus lors des recherches faites en Bulgarie. Bull. Off. Int. Epiz., 1957, 57 : 66-73.

48. PETROVIC (K.). - (Nous n'avons lu quiun abstrait dans :) Vet. Bull., 1959, $29: 11$.

49. PETROVIC (Z.), GOLOSIN (R.) et CVETKOVIC (A.). - (Nous n'avons lu qu'un abstrait dans:) Vet. Bull, 1960, 30:681.

50. PIERCY (P. L.). - Transmission of anaplasmosis. Ann. N. Y. Acad, Sci., 1956, 64 : 40-48.

51. POISSON $\left(H_{1}\right)$, — Prodrome d'études de parasitologie malgache. Etudes du Laboratoire de Recherches du Service Vétérınaire de Tananarive, 1927, $1: 12-18$.

52. POISSON (H.). - Sur un cas de gale psoroptique du bouf. Communication à la Soc. Sci. Méd. Madagascar, le 18 juın 1930.

53. POISSON $\left\{\mathrm{H}_{\text {. }}\right.$. - Les maladies parasitaires à Madagascar. Revue scientifique illustrée, 1931, $69: 230-237$.

54. PORTE (L.), CAPRON (A.), SUREAU (P.) et DERAN (C.). - A propos de la première observation clinique sérologiquement confirmée de fièvre $Q$ à Madagascar. Bull. Soc. Path. exot., 1959, 78-82.

55. RAPPORTS ANNUELS du Laboratare Central de I'Elevage, Tananarive, années 1929 à 1962.

56. RAYNAUD (J. P.). - Prospection des hématozoaires ef tiques de bovins à Madagascar. I. Recherches dans la province de Tananarive. Rev. Elev. Méd. vét. Pays trop., 1962, 15: 137-154.

57. RAYNAUD (J. P.). - Morphologie, chimiosensibilité ef réactions immunitaires de souches de Babesia bigemina (Smith et Kilborne 1893) mises en évidence par splénectomie de bov|ns. Rev. Elev. Méd, vét. Pays trop., 1962, $15: 167-179$.

58. RAYNAUD (J. P.). - Splénectomie des bovins et parasites sanguins. Ann. de Parasitologie, 1962, $37:$ :755-766.

59. RAYNAUD (J, P.) et UILENBERG (G.). Prospection des hématozoaires et tiques de bovins à Madagascar. II. Recherches complémentaires ef conclusions. Rev. Elev. Méd. vét. Pays trop., 1962, $15:$ 147-153.

60. REICHENOW (E.). - Lehrbuch der Protozoenkunde. 1953. 6e Edition. lena, Gustav Fischer Verlag.

61. RIEK (R. F.). - Rapport de la deuxième Réunion du Groupe d'Experts F.A. O./O.I. E. sur les maladies du bétail transmises par des tiques. Le Caire : 1962, 39-43.

62. SERGENT (E.), DONATIEN (A.), PARROT (L.) et LESTOQUARD (F.). - Etudes sur les piroplasmoses bovines. Institut Pasteur d'Algérie, Alger, 1945.

63. SIMITCH (T.) ef NEVENITCH (V.), - Babestella bovis (Babes, 1888) et Babesiella berbera Sergent, Donatien, Parrot, Plantureux et Rougebief, sont-ils synonymes? Arch. Inst. Pasteur d'Algérie, 1953, 31 :91-101.

64. SIMITCH (T.), PETROVITCH (Z.) ef RAKOVEC (R.). - Les espèces de Babesiella du bœuf d'Europe. Arch. inst. Pasteur d'Algérie, 1955, $33:$ 310-314.

65. SUREAU (P.). - Infection spontanée des souris d'élevage à Tananarive par Encephalitozoon cuniculi et Klosiella muris. Arch. Inst. Pasteur de Madagascar, 1963, 31 : 125-126.

66. SUREAU (P.), RAYNAUD (3. P.), LAPEIRE (C.) et BRYGOO (E. R.). - Premier isolement de Toxoplasma gondii à Madagascar. Toxoplasmose spontanée et expérimentale du Lemur catta. Buil.' 'Soc. Path. exot., 1962, 55 : 357-362.

67. SUREAU (P.) ef UILENBERG (G.). - Isolement à partir d'un pigeon domestique (Columba livio) d'une seconde souche de Toxoplasma gundii à Madagascar. Arch. Inst. Pasteur de Madagascar, 1963, 32 : 47-53.

68. THEILER (A.) - Gall-sickness of imported cattle and the protective inoculation against this disease. South African Agricultural Journal (janvier 1912) ; Tiré à part no 6, 1912 du Dept. of Agriculture, Union of South Africa, 23 pages.

69. THEILER (G.). - Notes on the ticks of domestic stock from Portuguese East Africa. Estaçao Anti-Malarica de Lourenço Marques. 1943. Imprensa Nacional de Moçambique. 
70. THEILER (G.). - Zoological survey of the Union of South Africa. Tick survey, part IX. The distribution of the three South African Hyalommas or bontpoots. Onderstep. J. Vet. Res., 1956, 27 : 239-269.

71. THEILER (G.) et SALISBURY (L. E.). - Zoological survey of the Union of South Africa. Tick survey : Part Xl. The distribution of Otobius megnini, the spinose ear tick. Onderstep. J. Vet. Res., 1958, $27:$ 605-610.

72. TSUR (1.). - Immunization trials against bovin babesiasis. I. Vaccination with blood from latent carriers. Refuah Veterinarith, 1961, $18: 110-103$.

73. TSUR (I.) et LAPINSKI (Z.). - Immunization trials against bovine babesiasis II. Vaccination with blood from «patent» carriers. Refuah Veterinarith 1962, 19 :183-181.
74. TSUR-TCHERNOMORETZ (I), - Blood parasites in lıvestock in Israel. Refuah Veterınarith, Israel 10 th Anniversary Issue (mai): 1959, 20-24.

75. UILENBERG (G.). - Boophilus (Uroboophilus) fallax Minning, 1934, synonyme de Boophilus microplus (Canestrini, 1887) (lxodidae). Rev. Elev. Méd. vét. Pays trop., 1962, 15 : 387-398.

78. UILENBERG(G.). - Existence de Ornithodorus porcinus Walton, 1962 (Argasidae) à Madagascar. Rev. Elev. Méd. vét. Pays trop., 1963, $16: 147-150$.

79. WALTON (G. A.), - The Ornithodorus moubato superspecies problem in relation to human relapsing fever epidemiology. Aspects of Disease Transmission by Ticks. Symposia of the Zoological Society of London. 1962. $n^{\circ} 6: 83-156$. 